\title{
Capítulo VI - Despliegue óptimo de redes PON, usando mapas reales, para un gran número de usuarios con diversas demandas de ancho de banda
}

\author{
Germán Arévalo \\ Roberto Gaudino
}

\section{SciELO Books / SciELO Livros / SciELO Libros}

ARÉVALO, G., and GAUDINO, R. Despliegue óptimo de redes PON, usando mapas reales, para un gran número de usuarios con diversas demandas de ancho de banda. In: INGA ORTEGA, E., ed. Aplicaciones e innovación de la ingeniería en ciencia y tecnología [online]. Quito: Editorial AbyaYala, 2019, pp. 201-232. ISBN: 978-9978-10-491-0. https://doi.org/10.7476/9789978104910.0008.

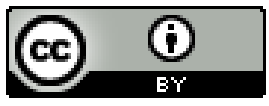

All the contents of this work, except where otherwise noted, is licensed under a Creative Commons Attribution 4.0 International license.

Todo o conteúdo deste trabalho, exceto quando houver ressalva, é publicado sob a licença Creative Commons Atribição 4.0.

Todo el contenido de esta obra, excepto donde se indique lo contrario, está bajo licencia de la licencia $\underline{\text { Creative }}$ Commons Reconocimento 4.0. 


\title{
CAPÍTULO VI
}

\section{Despliegue óptimo de redes PON, usando mapas reales, para un gran número de usuarios con diversas demandas de ancho de banda}

\section{Optimal deployment of PON, using real maps, for a large number of users with heterogeneous bandwidth demands}

\author{
Germán Arévalo , Roberto Gaudino \\ Universidad Politécnica Salesiana Ecuador, Politécnico Di Torino Italia \\ *Email: garevalo@ups.edu.ec \\ §Email: roberto.gaudino@polito.it
}

\section{Resumen}

El problema relacionado con la búsqueda de topologías óptimas para despliegue de PON en una ciudad, para servir a un determinado número de usuarios, es en esencia un problema del tipo Optimización del Árbol de Steiner, el cual es un bien conocido problema del tipo NP-Hard. Así, para proponer soluciones para este tipo de problemas se deben emplear procesos de tipo heurístico. Por otro lado, se tiene el problema de seleccionar la tecnología PON más apropiada para satisfacer las demandas de ancho de banda de los usuarios, con escalabilidad e inversión redituable. En el presente artículo se detalla los aspectos técnicos y resultados obtenidos a través de un algoritmo para el despliegue óptimo de redes PON en grandes regiones urbanas caracterizadas por un gran número de usuarios potenciales de tipo residencial y corporativo que demandan diversos anchos de banda. El estudio presentado incluye una comparación del costo de implementación de distintos tipos y estándares de redes PON.

\section{Palabras clave}

PON, estudio tecno-económico, optimización, algoritmo, heurísticas. 


\begin{abstract}
The problem related with the optimal search of topologies for the deployment of PON in an urban region, for servicing a given number of users, is essentially a Steiner-tree problem, which is a well-known NP-Hard problem. Therefore, in order to find solutions for this type of problems it is necessary the use of heuristic methods. On the other hand, there is the problem related with the selection of the most suitable PON technology, in order to satisfy the users' bandwidth demands in a scalable and profitable way. This paper details the technical aspects and the results obtained through the use of an algorithm which finds the optimal PON deployment in large urban regions with very large number of users with heterogeneous bitrate demands. The study covers the costs comparison among different types of PON standards.
\end{abstract}

\title{
Keywords
}

PON, techno-economics, optimization, algorithm, heuristics.

\section{Introducción}

Debido a los múltiples problemas confrontados en el desarrollo de redes ópticas de acceso de nueva generación (NG), el desarrollo de soluciones que viabilicen el despliegue comercialmente viable de este tipo de redes, a constituido un tópico de investigación muy popular en los últimos años, especialmente el estudio de las redes ópticas pasivas (PON) de nueva generación [1].

Particularmente, el estudio tecno-económico de las redes PON de nueva generación, mismas que incluyen en la tecnología de la red técnicas de transmisión y compartición del canal más complejas y avanzadas que las empleadas por los estándares actualmente existentes, como la multiplexación por división de longitud de onda (WDM) incluyendo la división de longitud de onda ultra-densa (UDWDM), ha recibido especial atención por parte de varios investigadores [2][3][4][5]. Las estrategias para la optimización este tipo de redes se basan en el estudio del despliegue de la red de distribución óptica (ODN) con el menor costo posible [6] [7][8], o en el diseño del hardware de la red [9][10].

En el año 2015 ya se publicó el estándar UIT para las redes ópticas pasivas de nueva generación, el Next-Generation Passive Optical Network - Version 2 (NGPON2) [11]. 
Se han propuesto varias alternativas para la topología de una red óptica de acceso, sin embargo la topología tipo árbol de PON es la única actualmente existente y comercialmente implementada en el mundo y que ha sido estandarizada para este tipo de redes [12]. La topología tipo árbol se basa exclusivamente en el empleo de divisores de potencia ópticos (splitters) en cascada con varias etapas, aunque en la práctica las PON se implementan hasta con dos niveles de splitting. En la Figura 1 se ilustra la topología lógica de una PON, donde puede observarse que la red parte de un transceptor óptico denominado "terminal de línea óptica" (OLT), ubicado en la "oficina central" (CO) del proveedor de servicios. A partir de ahí, a través de una fibra óptica (OF) denominada "fibra de alimentación" (Feeder) se arriba a un splitter remoto generalmente ubicado a varios kilómetros de distancia, dentro de un gabinete que generalmente se encuentra en una esquina sobre la vereda o en algún lugar de acceso público, al que se le denomina "gabinete de calle primario" (PSC). Desde ahí, a través de cables ópticos de distribución de menor longitud se llega o directamente al usuario final o a un segundo splitter para luego con cables ópticos de corta distancia (si el segundo splitter está en la entrada de un edificio, estos últimos cables generalmente con cables para interiores). En el extremo del usuario final se encuentra un equipo de transmisión-recepción denominado la "unidad óptica de red" (ONU). La red óptica que parte desde la OLT y llega a la ONU constituye la ODN.

Sin embargo, en la práctica, para cubrir a un gran número de usuarios se realiza un despliegue simultáneo de múltiples redes PON, desde varias CO con muchas OLT (colocadas en racks de OLT), compartiendo de manera parcial las rutas y cables que se despliegan y en consecuencia la infraestructura de la instalación (i.e. ductos, zanjas, cables subterráneos y aéreos, gabinetes de calle, etc.). En la Figura 2 se puede observar la topología física típica de una implementación real de múltiples PON en una región urbana. 


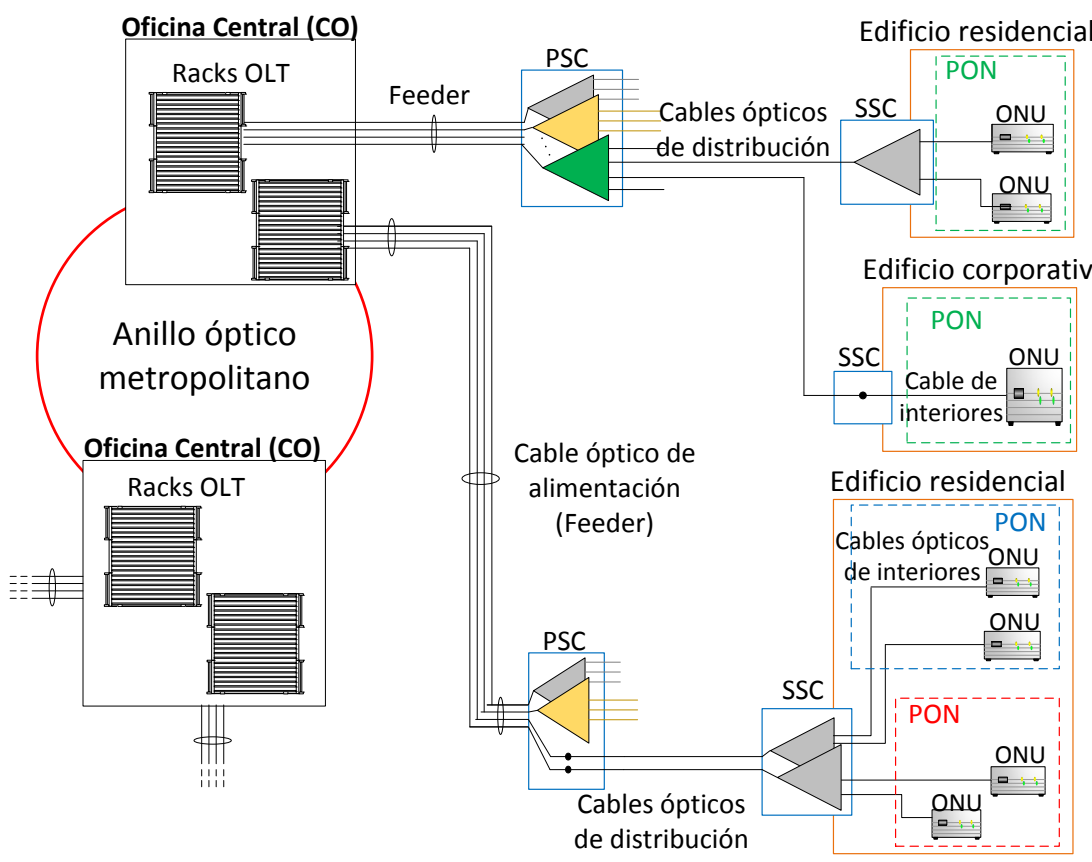

Figura 1. Topología lógica y nomenclatura de una PON

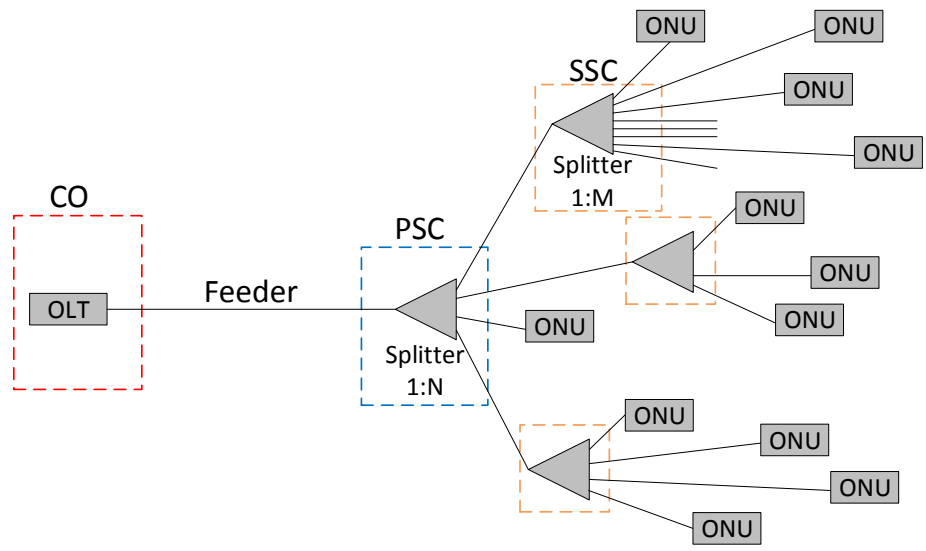

Figura 2. Topología física típica de un despliegue de múltiples PON en una región urbana con muchos usuarios 
Dada la complejidad que implica la instalación de este tipo de topologías, el estudio de la planificación para el depliegue óptimo de PON es de mucho interés para los operadores [13].

El resto del presente artículo está organizado como sigue: La sección 2 describe los parámetros generales, tanto de los escenarios urbanos a ser considerados, como los costos referenciales y parámetros técnicos de los distintos tipos de PON que se cubren en este estudio. En la sección 3 se presentan los detalles de la formulación del problema de optimización que modela la búsqueda del despliegue de menor costo para múltiples PON en un escenario urbano extenso. La sección 4 explica los detalles del algoritmo matemático desarrollado para la solución del problema. En la sección 5 se muestran los resultados más relevantes de la investigación y, finalmente la sección 6 concluye el artículo.

\section{Parámetros y variables del problema}

\section{A. Consideraciones generales}

El presente estudio se enfoca en el despliegue de redes PON para proveer fibra óptica al hogar (FTTH) a través de un óptimo dimensionamiento y planeación de la ODN.

Para tal fin se consideran tres tipos de PON estandarizadas: (i) la GPON, (ii) la XGPON, [19] mismas que pueden conectar hasta 64 usuarios empleando TDM/TDMA como técnicas de compartición del canal para bajada "downstream - DS" y subida "upstream - US", respectivamente, y (iii) el nuevo estándar NGPON2 [11], introducido por primera vez en el 2013 y cuya última revisión se publicó en el 2015, misma que es una red híbrida TDM/WDM con entre cuatro a ocho canales WDM densos (DWDM) tanto para DS como para US. NGPON2 no reemplaza los splitters por multiplexores, sino los mantiene con el fin de proveer compatibilidad con la ODN de estándares anteriores de redes PON.

Algunos trabajos de información relevantes proponen soluciones de hardware de bajo costo para NGPON2, como el reportado en [20]. También usamos en nuestro análisis una propuesta de red de 
nueva generación a largo plazo denominada UDWDM-PON (PON con multiplexación ultra-densa de longitudes de onda) [21].

El escenario que se emplea para el desarrollo del presente estudio es el de una región urbana (i.e. una zona dentro de una ciudad o urbe) en la cual existe una gran densidad de habitantes y de entre ellos un gran número de usuarios (en el orden de cientos de miles) demandan servicios de acceso a Internet, con distintos anchos de banda, a través de conexiones FTTH. Tal número de usuarios no podrían ser cubiertos por una sola $\mathrm{CO}$, de modo que se considera la presencia de varias $\mathrm{CO}$ en la región, cada una de ellas cubriendo una subregión de hasta aproximadamente 20000 usuarios a través de varios cientos de OLT en sus racks (i.e. cada OLT significa una PON individual a ser desplegada en la ciudad). Se asume que las CO están interconectadas entre sí, por ejemplo, a través de un anillo óptico metropolitano, como el que se observa en la Figura 2.

\section{B. Costos referenciales}

A través de interacción directa con proveedores de servicios y vendedores de equipos, hemos obtenido la información de costos referenciales que se detallan a continuación, en las Tablas 1, 2 y 3.

Tabla 1. Costos de cables ópticos y trabajos de instalación

\begin{tabular}{|c|c|}
\hline Componente & Costo (\$) \\
\hline Feeder Cable, 2 fibras $/ \mathrm{km}$ & 600 \\
\hline Feeder Cable, 4 fibras $/ \mathrm{km}$ & 1000 \\
\hline Feeder Cable, 6 fibras $/ \mathrm{km}$ & 1500 \\
\hline Feeder Cable, 12 fibras $/ \mathrm{km}$ & 2000 \\
\hline Feeder Cable, 24 fibras $/ \mathrm{km}$ & 2500 \\
\hline Feeder Cable, 48 fibras $/ \mathrm{km}$ & 3000 \\
\hline Feeder Cable, 64 fibras $/ \mathrm{km}$ & 3500 \\
\hline Feeder Cable, 96 fibras $/ \mathrm{km}$ & 3700 \\
\hline Feeder Cable, 140 fibras $/ \mathrm{km}$ & 4000 \\
\hline Feeder Cable, 280 fibras $/ \mathrm{km}$ & \\
\hline
\end{tabular}




\begin{tabular}{|c|c|}
\hline Componente & Costo (\$) \\
\hline Distribution Cable $/ \mathrm{km}$ & 2000 \\
\hline Cable de interiores /usuario & 50 \\
\hline Zanjado y recapeo/km & 30000 \\
\hline Ductos/km & 10000 \\
\hline Empalmes/unidad & 10 \\
\hline Pozos de revisión/unidad & 500 \\
\hline
\end{tabular}

Tabla 2. Costo de gabinetes

\begin{tabular}{|c|c|}
\hline Componente & Costo (\$) \\
\hline Caja de empalmado 140 OF & 400 \\
\hline Caja de empalmado 48 OF & 350 \\
\hline Caja de empalmado 16 OF & 300 \\
\hline Caja de empalmado 8 OF & 120 \\
\hline $1: 64$ splitter & 70 \\
\hline $1: 32$ splitter & 45 \\
\hline $1: 16$ splitter & 28 \\
\hline $1: 8$ splitter & 24 \\
\hline $1: 4$ splitter & 20 \\
\hline $1: 2$ splitter & 1600 \\
\hline Gabinete (incluida instalación) & \\
\hline
\end{tabular}

Tabla 3. Costos del hardware PON

\begin{tabular}{|c|c|}
\hline Componente & Costo $(\$)$ \\
\hline OLT chasis - GPON (103 usuarios) & 16000 \\
\hline OLT chasis - XGPON (103 usuarios) & 28000 \\
\hline OLT chasis - NGPON2 (103 usuarios) & 50000 \\
\hline OLT chasis - UDWDM PON (103 usuarios) & 85000 \\
\hline
\end{tabular}




\begin{tabular}{|c|c|}
\hline Componente & Costo $(\mathbf{\$})$ \\
\hline OLT tarjeta - 8xGPON & 9000 \\
\hline OLT tarjeta - 8xXGPON & 15000 \\
\hline OLT tarjeta - 8xNGPON2 & 25000 \\
\hline OLT tarjeta - 8xUDWDW-PON & 40000 \\
\hline ONU residencial - GPON & 100 \\
\hline ONU residencial - XGPON & 350 \\
\hline ONU residencial - NGPON2 & 600 \\
\hline ONU residencial - UDWDM PON & 1100 \\
\hline ONU corporativa - GPON & 350 \\
\hline ONU corporativa - XGPON & 600 \\
\hline ONU corporativa - NGPON2 & 1100 \\
\hline ONU corporativa - UDWDM PON & 2200 \\
\hline Fusión / por empalme & 10 \\
\hline Instalación de la OLT & 2000 \\
\hline ODF (para cada rack OLT) & 3500 \\
\hline
\end{tabular}

\section{Parámetros de la red}

En la Tabla 4 se especifican los parámetros generales de cada tecnología PON que se considera en este estudio. El enfoque de la investigación es en la demanda de ancho de banda de bajada, DS, por parte de los usuarios, dado que el tráfico DS por parte de los usuarios es mucho mayor que el US. En el caso de UDWDM PON, se emplean los parámetros de la propuesta de Rohde reportada en [22], con la única variación de que en lugar de los 1024 usuarios a 1 Gb/s que se pueden conectar con la propuesta de Rohde, en este trabajo se considera un número más conservativo: 256 usuarios por cada OLT.

Además, se consideran los siguientes:

- Tipo de OF: UIT G652 D (monomodo estándar) 
- Atenuación en los splitters: $\alpha=3,5 * \log _{2}(k) \mathrm{dB}$ [99] (donde $k$ es el número de ramales de salida del splitter).

- Bitrate referencial (para normalización): $\mathrm{BR}_{\text {ref }}=10 \mathrm{~Gb} / \mathrm{s}$.

En lo que respecta a las demandas de ancho de banda, o bitrate (BR), consideramos dos tipos de usuarios: residenciales y corporativos (donde el número de corporativos representan apenas el $2 \%$, con es lo usual). Las demandas se consideran del tipo "ancho de banda mínimo garantizado" y para simular un escenario realista, estas son seleccionadas aleatoriamente dentro de los seis posibles rangos especificados en la Tabla 5.

Tabla 4. Parámetros de las tecnologías PON consideradas

\begin{tabular}{|c|c|c|c|c|}
\hline \multirow{2}{*}{ Componente } & \multicolumn{4}{|c|}{ Tecnología PON } \\
\cline { 2 - 5 } & GPON & XGPON & NGPON2 & UDWDM PON \\
\hline Max. longitud de enlace [km] & 40 & 40 & 40 & 100 \\
\hline Max. atenuación de ODN[dB] & 35 & 35 & 35 & 43 \\
\hline Usuarios por cada OLT & 64 & 64 & 64 & 256 \\
\hline Num. de longitudes de onda & 1 & 1 & 4 & 256 \\
\hline DS bitrate por OLT [Gb/s] & 2.5 & 10 & 40 & 256 \\
\hline
\end{tabular}

Tabla 5. Escenarios de demanda de bitrate

\begin{tabular}{|c|c|c|}
\hline \multirow{2}{*}{ Escenario } & \multicolumn{2}{|c|}{ Bitrate $(\mathbf{M b} / \mathbf{s})$ por tipo de usuario } \\
\cline { 2 - 3 } & Residencial & Corporativo \\
\hline 1 & $10-50$ & $100-500$ \\
\hline 2 & $50-100$ & $500-1000$ \\
\hline 3 & $100-400$ & $1000-2500$ \\
\hline 4 & $100-1000$ & $1000-10000$ \\
\hline 5 & $500-2500$ & $2500-10000$ \\
\hline 6 & $1000-2500$ & $5000-40000$ \\
\hline
\end{tabular}

\section{Formulación del problema}

\section{A. Definición de conjuntos y variables}

Una región urbana donde se requiere el despliegue de una serie de redes PON, con el fin proveer acceso a la red a un determinado número de usuarios aleatoriamente localizados dentro de esa 
región, puede ser tratado como un "grafo de conexión con pesos" (i.e. con conexiones dimensionadas de acuerdo a algún parámetro que permite determinar su "costo" o "peso"). En dicho grafo, las calles de la ciudad y sus intersecciones constituyen los bordes y los nodos del grafo, mismos que pueden ser utilizados como rutas de conexión desde las OLT, hasta las ONU, pasando por sus correspondientes PSC y SSC.

Para describir matemáticamente el problema de optimización que modela la búsqueda del despliegue de redes PON en una ciudad al menor costo posible, es necesario primero definir las siguientes constantes, variables y conjuntos:

- CO: El conjunto de oficinas centrales, $C O=\left\{C O_{C}\right\}$, con $c \in\{1,2, \ldots, C\}$; donde $C$ es el número de $C O$ disponibles.

- $\quad N_{C}$ : El número de usuarios atendidos por la oficina central $c$, de manera que $\Sigma N_{C}=N$, donde $N$ es el número total de usuarios en la región.

- $\quad O$ : El conjunto de $O L T, O=\left\{O_{O}\right\}$, con $o \in\{1,2, \ldots, M\}$, donde $M$ es el número de OLT disponibles.

- $\quad U$ : El conjunto de $O N U, U=\left\{O N U_{n}\right\}$, con $n \in\{1,2, \ldots, N\}$, donde $N$ es el número de $O N U$ requeridas.

- $W$ : El conjunto de longitudes de onda, $W=\{\lambda w\}$, con $w \in\{1$, $2, \ldots, L\}$; donde $L$ es el número de longitudes de onda disponibles en el transmisor de una OLT. Específicamente: $L=1$ para $G P O N$ y $X G P O N, L=4$ para $N G P O N 2$ y $L=256$ para $U D W D M$ $P O N$.

- $\quad L_{i}$ : El conjunto de splitters disponibles en un gabinete localizado en el sitio $i$. También se define en este contexto al parámetro $S_{i, l}$ como el lésimo splitter, en el gabinete $i$, cuyo rango de división ("splitting ratio" - $S R$ ) se denota como $K_{i, l}$ y es igual a $2^{r}$, donde $r$ es número entero positivo.

- $B$ : Es el conjunto de sitios candidatos para colocar un SSC.

- $\quad V$ : Es el conjunto de sitios candidatos para colocar un PSC.

- $n_{\max }$ : Es el máximo número de usuarios por OLT.

- $O D N_{\text {loss }}$ : La máxima atenuación, en $d B$, permitida en la $O D N$. 
Parámetros relacionados con la capacidad de la red PON se definen como sigue:

- $\quad B R_{\text {ref }}$ : Bitrate de referencia (para normalización).

- $B R_{U S / \lambda}$ : La bitrate total para US por OLT y por cada $\lambda$.

- $B R_{D S / \lambda}$ : La bitrate total para $D S$ por OLT y por cada $\lambda$.

- $B R_{U S}^{n}$ : La bitrate total demandada por la ONU $n \in U c$ en dirección US.

- $\quad B R_{D S}^{n}$ : La bitrate total demandada por la $O N U n \in U c$ en dirección $D S$.

- $\quad T_{U S}$ : La capacidad total normalizada de bitrate en dirección US para una $O L T$, tal que $T_{U S}=\left(L \cdot B R_{U S / \lambda}\right) / B R_{r e f}$

- $T_{D S}$ : La capacidad total normalizada de bitrate en dirección $D S$ para una OLT, tal que $T_{D S}=\left(\mathrm{L} \cdot \mathrm{BR}_{\mathrm{DS} / \lambda}\right) / B R_{\text {ref }}$

- $\quad \gamma_{U S}^{n}$ : La demanda de bitrate normalizada de la $O N U n \in U c$, en dirección $D S$, tal que $\gamma_{U S}^{n}=B R_{U S}^{n} / B R_{r e f}^{n}$

- $\quad \gamma_{D S}$ La demanda de bitrate normalizada de la ONU $n \in U c$, en dirección DS, tal que $\gamma_{D S}^{n}=B R_{D S}^{n} / B R_{\text {ref }}$

Además, se empleará un conjunto de parámetros relacionados con los sitios, rutas físicas y costos. Para el efecto se supondrá que en la región bajo estudio ST es un conjunto de calles apropiadas para enrutar cables ópticos y BL es el conjunto de edificios (i.e. cualquier lugar donde haya uno o más usuarios demandando conectividad):

- $\quad$ : El conjunto de nodos en calles y en edificios (intersecciones y vértices), $\mathrm{I}=\{i \in\{S T, B L\} / i=1,2, \ldots, T\}$; donde $T$ es el número total de nodos.

- $E$ : El conjunto de bordes (enlaces entre nodos) $E=\left\{e_{i, j} \in E /\right.$ $(i, j) \in I\}$.

- $\quad \alpha_{o}^{\mathrm{c}}$ : es una constante binaria que indica (si su valor se 1) que la $O L T$ O $\in O$ está ubicada en la oficina central $c \in C O$.

- $d_{i, j}$ La distancia total entre dos nodos $(i, j) \in I$ no necesariamente adyacentes.

- $\quad C_{O F}^{F}$ : Es el costo por unidad de longitud de un cable óptico de alimentación (Feeder OF).

- $C_{O F}^{D}$ : Es el costo por unidad de longitud de un cable óptico de distribución (Distribution OF). 
- $C_{T}$ : El costo por unidad de longitud del zanjado

- $C_{\text {encl }}^{i}:$ El costo de un gabinete óptico en el sitio $i$

- $C_{i, l}$ El costo del lésimo splitter en el gabinete $i$.

- $C_{O L T}^{\text {rack, }}$ : El costo de un rack de OLTs con capacidad total de hasta $\eta$ usuarios.

- $C_{\text {OLT }}^{\text {card }}$ El costo de un tranceiver OLTs.

- $C_{i, l}$ El costo del lésimo splitter en el gabinete $i$.

- $C_{O D F}$ : El costo de un gabinete de distribución óptico (ODF).

- $C_{O N U}$ El costo de una ONU.

- $C_{l b r}^{c}$ : El costo de las labores de instalación y configuración del hardware $P O N$ en la oficina central $c$.

- $\alpha_{O F}$ : La máxima por unidad de longitud en la $O F$.

- $\alpha_{i, 1}$ Atenuación en el lésimo splitter ubicado en el gabinete $i$.

- $\alpha_{e x}$ La atenuación insertada en la ODN por otras fuentes.

Adicionalmente, el modelo de optimización propuesto en este trabajo requiere la definición de las siguientes variables binarias:

$$
\begin{aligned}
& x_{n, j}= \begin{cases}1, & \text { si la ONU } n \text { está conectada al SSC } j \\
0, & \text { de otra manera }\end{cases} \\
& x_{j, i}=\{1 \text {, si el SSC } j \text { está conectada al PSC } i \\
& x_{i, o}=\left\{\begin{array}{l}
1, \text { si un splitter en el PSC } i \text { está conectado a la OLT } o \\
0, \text { de otra manera }
\end{array}\right. \\
& \alpha_{i}= \begin{cases}1, & \text { si el sitio candidato } i \in\{V \cup B\} \text { está activo } \\
0, & \text { de otra manera }\end{cases} \\
& \alpha_{o}= \begin{cases}1, & \text { si la OLT } o \text { está activa (conectada) } \\
0, & \text { de otra manera }\end{cases} \\
& S_{i, l}= \begin{cases}1, & \text { si el } l^{\text {ésimo }} \text { splitter en el sitio } i \text { está conectado } \\
0, & \text { de otra manera }\end{cases} \\
& y_{n}^{j, l}= \begin{cases}1, & \text { si la ONU } n \text { se conecta al el } l^{\text {ésimo }} \text { splitter } i \\
0, & \text { de otra manera }\end{cases} \\
& y_{j, l}^{i, p}=\left\{\begin{array}{l}
1, \text { si el } l^{\text {ésimo }} \text { splitter ubicado en el sitio } j \text { se } \\
\text { conecta con el } p^{\text {ésimo }} \text { splitter del sitio } i \\
0, \text { de otra manera }
\end{array}\right. \\
& z_{n}^{o}= \begin{cases}1, & \text { si la ONU } n \text { se conecta con la OLT } o \\
0, & \text { de otra manera }\end{cases}
\end{aligned}
$$




\section{B. Formulación del problema de optimización}

La función objetivo del problema de optimización apunta a minimizar el costo total de implementación de las múltiples PON que deberán ser desplegadas a partir de varias $\mathrm{CO}$ en una gran zona urbana extensa con gran número de usuarios en ella. Una ventaja de optimizar toda la extensa zona en el mismo problema es que se puede variar la distribución de las subregiones servidas por cada CO con el fin de buscar la resolución óptima del problema. En la siguiente sección explicaré cómo se resolvió a través de un enfoque heurístico este problema combinatorio. En media se tendrá N / C usuarios en cada subregión servida por las $\mathrm{CO}$ ( $\mathrm{N}$ es el número total de usuarios y C el número de $\mathrm{CO}$ en la región extensa a ser servida).

La función objetivo es la que se muestra en la Ecuación (1).

$$
\begin{aligned}
\min \sum_{c \in C O}\left(C_{l b r}^{c}+\right. & C_{T}\left(\sum_{o \in O} \sum_{i \in V} \alpha_{o}^{c} x_{i, o} d_{i, o}+\sum_{i \in V} \sum_{j \in B} \alpha_{o}^{c} x_{j, i} d_{j, i}\right) \\
& +C_{O F}^{f} \sum_{o \in O} \sum_{i \in V} \alpha_{o}^{c} x_{i, o} d_{i, o}+\sum_{i \epsilon\{V U B\}} C_{O L T}^{c a r d} \alpha_{o} \\
& +C_{O F}^{d}\left(\sum_{i \in V} \sum_{j \in B} x_{j, i} d_{j, i}+\sum_{j \in B} \sum_{n \in U} x_{n, j} d_{n, j}\right) \\
& +\sum_{i \in\{V U B\}} \sum_{l \in L_{i}} S_{i, l} C_{i, l}+\sum_{i \in\{V U B\}} C_{e n c l}^{r} \alpha_{i} \\
& \left.+\frac{N}{\eta}\left(C_{O L T}^{r a c k, \eta}+C_{O D F}\right)+C_{O N U} N\right)
\end{aligned}
$$

La ecuación (1) está compuesta por una suma global que calcula el costo de implementación para cada subregión servida por las CO. La suma de costos incluye, respectivamente: los costos de labor en la $\mathrm{CO}$ y de despliegue de la OF tanto para el zanjado como para el costo de los propios cables, el costo del hardware PON en la CO, el costo de los splitters y gabinetes de calle, y el costo de las ONU. 
El conjunto de restricciones que aseguran que el modelo esté apegado a una implementación realista de la red son:

- El número de usuarios conectados a la CO debe ser igual a:

$$
N_{C}=\sum_{n \in U} \sum_{o \in O} \alpha_{o}^{c} z_{n}^{o} ; \quad \forall c \in C O
$$

- El número de usuarios por OLT debe ser menor o igual a:

$$
\sum_{n \in U} z_{n}^{o} \leq n_{\max } \alpha_{o} ; \quad \forall c \in C O
$$

- La demanda de bitrate máxima por OLT no debe ser mayor que su capacidad US y DS:

$$
\sum_{n \in U} z_{n}^{o} \gamma_{U S / D S}^{n} \leq \Gamma_{U S / D S} \alpha_{o} ; \quad \forall o \in O
$$

- Una ONU debe estar conectada a un solo splitter que esté ubicado en un determinado sitio $\mathrm{j}$ :

$$
\sum_{n \in U} x_{n, j}=1 ; \quad \forall n \in U
$$

- Un SSC debe estar conectado a un y solo un PSC:

$$
\sum_{i \in V} x_{j, i}=\alpha_{j} ; \quad \forall j \in B
$$

- Un splitter en un PSC debe llegar a una y solo una OLT:

$$
\sum_{o \in O} x_{i, o} S_{i, l}=\alpha_{i} ; \quad \forall i \in V
$$


- El número de splitters activos en un sitio i debe ser menor o igual a la capacidad del gabinete:

$$
\sum_{n \in U} s_{i, l} \leq \alpha_{i} r_{i} ; \quad \forall i \in\{V \mathrm{U} B\}
$$

- Una ONU se puede conectar a un splitter en el sitio i si existe una conexión física desde la ONU al sitio i.

$$
y_{n}^{i, l} \leq x_{n, i} ; \quad \forall i \in\{V \mathrm{U} B\}, \forall n \in U, \forall l \in L_{i}
$$

- Un splitter 1 en un SSC ubicado en el sitio j se puede conectar con otro splitter p en un PSC ubicado en el sitio i, si existe una conexión física desde i a j.

$$
y_{j, l}^{i, p} \leq x_{j, i} ; \quad \forall i \in V, \forall p \in L_{i}, j \in B, \forall l \in L_{j}
$$

- $\quad$ El número de ONU que se conectan al lesimo splitter activo de un SSC en el sitio j no puede exceder la capacidad del splitter.

$$
\sum_{n \in U} y_{n}^{j, l} \leq K_{j, l} S_{j, l} ; \quad \forall j \in B, \forall l \in L_{j}
$$

- El número de ONU más splitters secundarios que se conectan a un splitter primario, no puede exceder la capacidad del splitter

$$
\sum_{n \in U} y_{n}^{j, p}+\sum_{j \in B} \sum_{l \in L_{i}} y_{j, l}^{j, p} \leq K_{i, p} S_{i, p} ; \quad \forall i \in V, \forall p \in L_{i}
$$

- La atenuación desde una ONU a su respectiva OLT debe ser menor o igual a la máxima atenuación permitida en la ODN:

$$
\begin{aligned}
& \alpha_{F o}\left(\sum_{j \in B} x_{n, j} d_{n, j}+\sum_{i \in V} \sum_{j \in B} \sum_{o \in O} x_{n, j} x_{i, o} x_{j, i} d_{j, i}+\sum_{i \in V} \sum_{o \in O} x_{i, o} d_{j, o}\right) \\
& +\sum_{j \in B} \sum_{l \in L_{j}} y_{n}^{j, l} S_{j, l} \alpha_{j, l}+\sum_{i \in V} \sum_{p \in L_{i}} \sum_{j \in B} \sum_{l \in L_{j}} y_{n}^{j, l} y_{j, l}^{i, p} S_{i, p} \alpha_{i, p} \\
& +\alpha_{e x} \leq \mathrm{ODN}_{\text {loss }}
\end{aligned}
$$




\section{Algoritmo de Solución Desarrollado}

\section{A. Función primaria de OTS}

El problema descrito por la Ecuación (1), se trata de un problema de optimización del tipo Árbol Mínimo de Steiner, el cual es un bien conocido problema del tipo NP-hard, lo que significa que a medida que no tiene solución exacta o no se puede calcular en un tiempo finito [23]. De modo que para encontrar una solución he desarrollado un algoritmo que hace uso de una serie de heurísticas. El algoritmo trabaja con una función principal (PF) y una serie de funciones secundarias (SF) que son ejecutadas programadamente para que realicen una determinada tarea. A este algoritmo lo he denominado Optimal Topology Search (OTS) [18], mismo que trabaja usando datos de mapas reales provistos por la plataforma de uso abierto OpenStreetMaps $^{\mathrm{TM}}$ (OSM). Esto le da la capacidad a OTS de resolver problemas de prácticamente cualquier lugar en el mundo.

La PF carga los datos de OSM (i.e. las coordenadas de estructuras pertenecientes a calles y edificios, incluyendo sus atributos). Dicha información es utilizada tanto para graficar el mapa de la zona donde se realiza el despliegue de la fibra, como para los cálculos que realiza el algoritmo.

En el mapa descargado, cuando se usa el modo simulación (i.e. sin usar datos reales de clientes, su ubicación y demanda de bitrate), PF genera aleatoriamente usuarios en los edificios (dependiendo de su tamaño y tipo de edificio: residencial o corporativo) y les asigna aleatoriamente una demanda de ancho de banda, dependiendo del escenario que se quiere simular (ver Tabla 5). La Figura 3 ilustra una porción de mapa con usuarios.

La generación aleatoria de usuarios se hace en base al área de los edificios (que se puede calcular en base a las coordenadas de su perfil) y al número de pisos de este (información proporcionada directamente por OSM).

OTS tiene la capacidad de resolver el problema para un gran número de usuarios (en el orden de los cientos de miles) todos ellos con heterogéneas demandas de ancho de banda. 


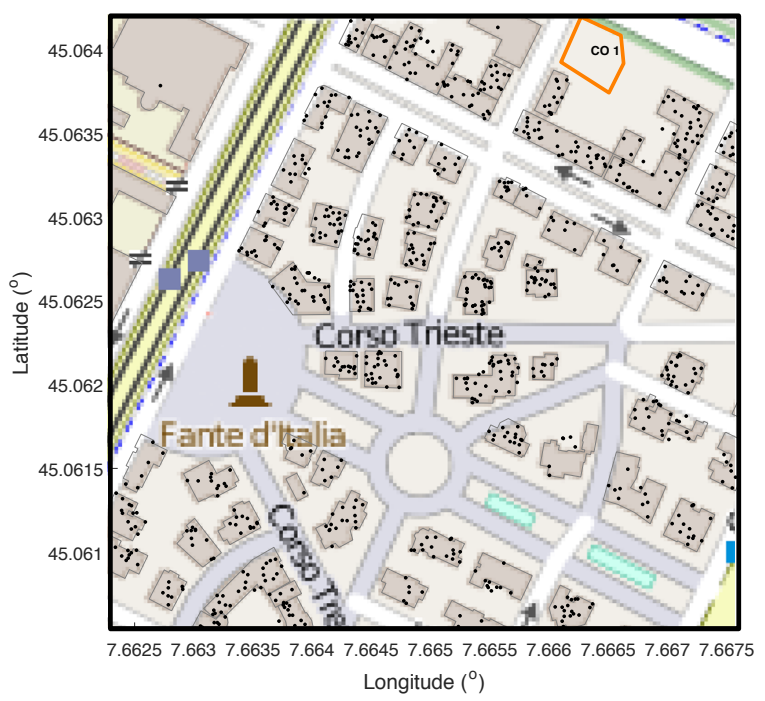

Figura 3. Mapa de ciudad con usuarios en edificios residenciales y corporativos (uno de este tipo se ve en la esquina superior izquierda) generados por OTS

A continuación PF agrupa los usuarios en grandes clúster alrededor de las CO a través de una teselación del tipo Voronoi de la región [24]. Cada clúster significa una subregión servida por su respectiva CO. Inicialmente el centro de cada clúster corresponde al centro de masa de los usuarios, el cual no corresponde (a menos que coincidencialmente coincida) a la ubicación del edificio de la CO. A través de las SF (que son escritas en detalle a continuación) se evalúa el costo del despliegue de múltiples PON usando esa cluterización inicial, y se guarda ese costo. Luego, se realiza una variación de los centros de los clúster (lo que significa un cambio de su tamaño y forma) en dirección a la ubicación geográfica de la CO, y se vuelve a calcular el costo. Este nuevo costo es comparado con el costo anterior, y si resulta mejor, se lo registra como el "menor costo" hasta ahora encontrado y se salva su topología, de otra manera se descarta. Así sucesivamente el algoritmo busca con iteraciones y finura programables el menor costo. El algoritmo converge luego de un determinado número de iteraciones que no se ha encontrado un costo menor (por defecto cinco, pero se puede cambiar ese valor 
para dar más confiabilidad, aunque eso aumenta la exigencia del cómputo). Dicho procedimiento constituye una heurística del tipo Tabu Search [25].

En la Figura 4 se ilustra un mapa con tres $\mathrm{CO}$ y sus respectivas subregiones iniciales (definidas por las líneas inter-punteadas de color negro) y posteriormente los mapas con clúster modificados, luego de varias iteraciones del algoritmo, donde los centros de masa de los clúster se han movido acercándose a la ubicación de los edificios de las CO.

\section{B. Funciones secundarias de OTS}

El conjunto de SF, llamadas por PF, son las siguientes:

- allocate_ssc: Identifica los edificios que tienen usuarios (residenciales o corporativos) y les asigna un SSC una acometida desde la calle más cercana al edificio (de entre todas las que circundan los mismos). En la Figura 5 se observa una región con varios edificios a los cuales la función allocate ssc les ha asignado un SSC (triángulos rojos) incluyendo su acometida (líneas de color rojo) perpendicular hasta la calle más cercana (las calles son resaltadas por las líneas de color azul en la gráfica).

- clustr_build: Divide una subregión de usuarios en clusters, pero no lo hace agrupando usuarios directamente sino a edificios. Se utiliza un enfoque del tipo Shared Nearest Neighbor (SNN) [26], en el cual los edificios son considerados como entidades individuales con atributos numéricos y categóricos. Esta función secundaria de OTS utiliza tres atributos específicamente: el tipo de usuarios en este, el número de usuarios, y el ancho de banda agregado que demandan los usuarios dentro del edificio. Así los usuarios dentro de los edificios terminan agrupados según los parámetros antes mencionados, lo cual es muy útil para dimensionar adecuadamente el hardware PON necesario para atender sus requerimientos de ancho de banda. 


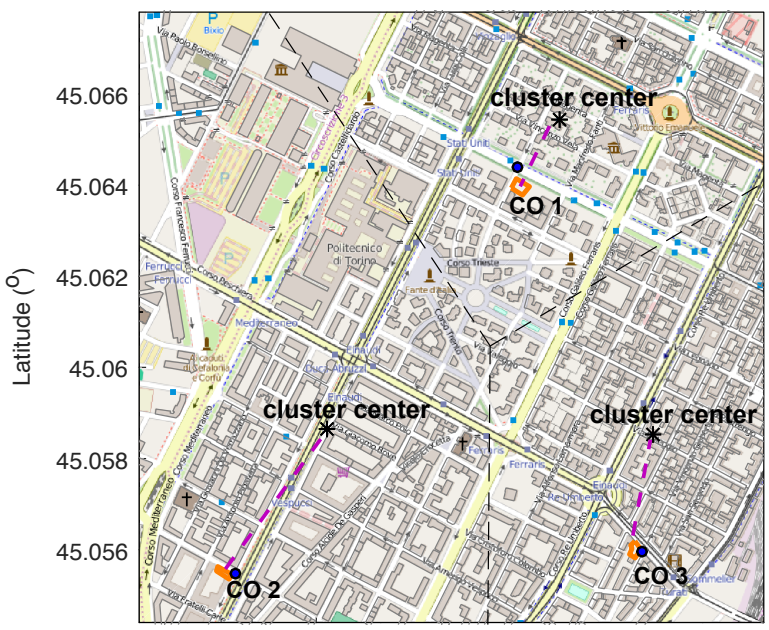

7.6567 .6587 .667 .6627 .6647 .6667 .6687 .677 .672

Longitude $\left({ }^{\circ}\right)$

(a)

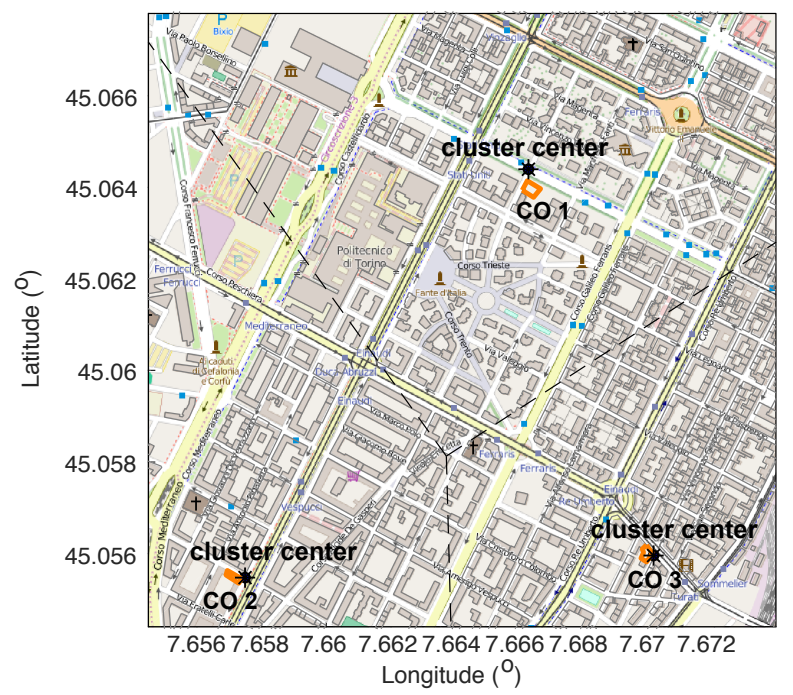

(b)

Figura 4. Ilustración del cambio de los las regiones servidas por las $\mathrm{CO}$, en la medida que OTS mueve su centro de masa en cada iteración. (a) Centros de los clúster lejos de los edificios de sus respectivas $\mathrm{CO}$, (b) Centros de los clúster cerca de los edificios de las $\mathrm{CO}$ 
En la agrupación se toma en cuenta la capacidad de la tecnología PON que se esté planificando desplegar (máximo ancho de banda y número máximo de usuarios que se puede servir con una OLT de la tecnología PON que se emplee). En la Figura 6 se observa la asignación de usuarios y asignación de los mismos a una determinada PON (a una OLT) a través de la agrupación de edificios (cada color corresponde a una PON). Este enfoque es realista dado que en la práctica no es eficiente conectar a la mitad de usuarios de un edificio a una OLT y la otra mitad a otra OLT.

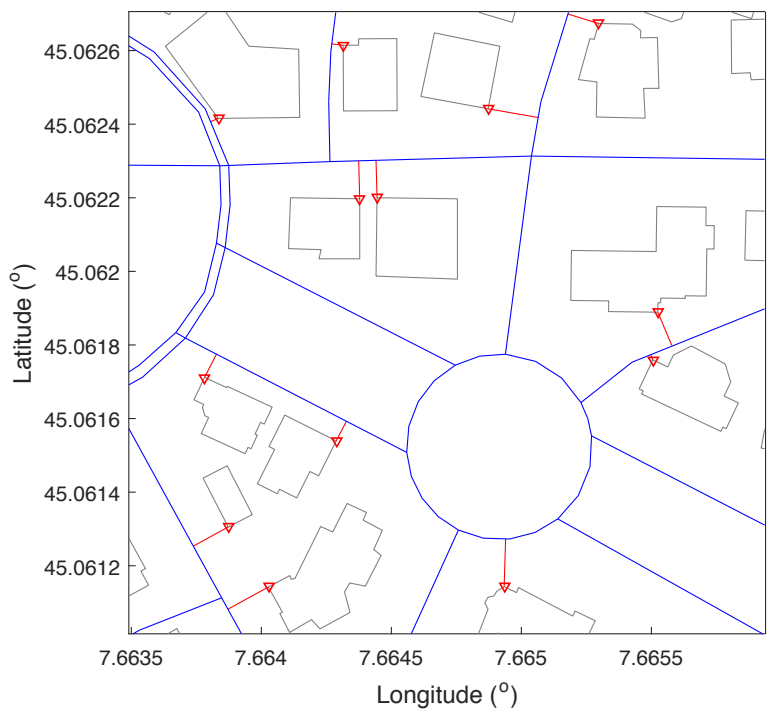

Figura 5. Ilustración de la asignación de SSC a edificios y acometida de OF a la calle más cercana

- aggregate: Dimensiona la cantidad y ubicación de los PSC en la ciudad a través de la clusterización de PON (las PON que fueron previamente dimensionadas y asignadas a usuarios por la función anterior). Para el efecto se utiliza una serie de sitios candidatos y nuevamente una teselación de Voronoi (esta vez dentro de la subregión servida por una sola CO). Los centros de masa de los clusters se emplean para la selección inicial del sitio para colocar el PSC, y a través de una búsqueda utilizando un 
algoritmo del tipo Random Search, que modifica la cantidad de PSC en cada iteración, se dimensiona de forma óptima los PSC en una subregión. La Figura 7 muestra la agrupación de PON a través de una teselación de Voronoi (líneas inter-punteadas de color negro) y la ubicación óptima de los PSC.

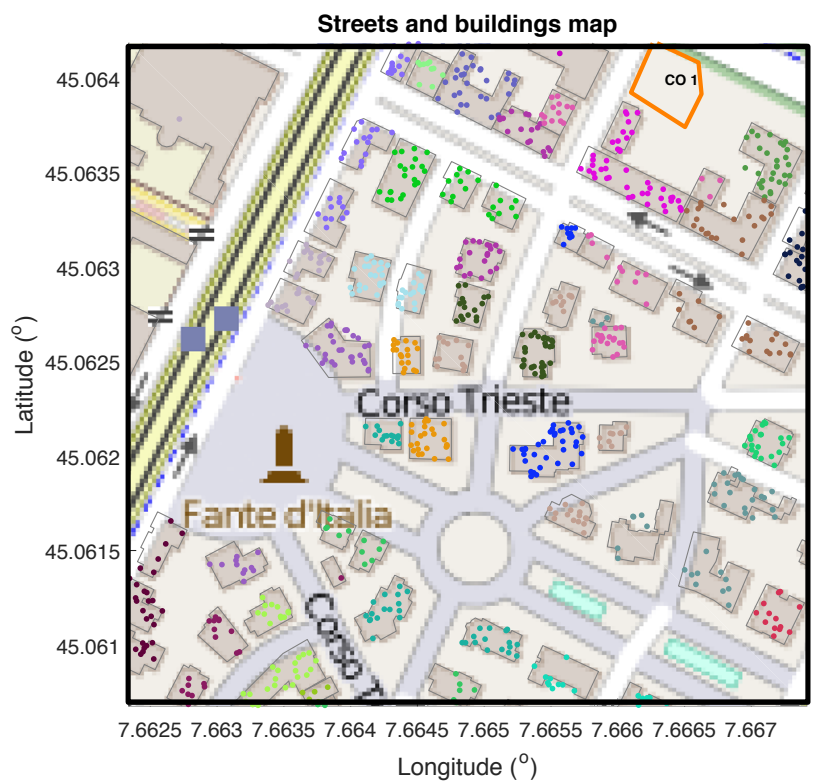

Figura 6. Clusters de usuarios para asignación de hardware PON. Cada color corresponde a un grupo de ONU de usuarios asignados a la misma OLT (cada color corresponde a una PON individual)

- OF_feeder;OF_distrb;Share: Estas tres funciones evalúan el zanjado, la compartición de ductos, y las rutas óptimas para el despliegue de los cables ópticos desde las CO hasta los usuarios finales. Para el efecto se utiliza una versión modificada del algoritmo de Dijkstra, el cual busca la iterativamente las rutas más cortas utilizando el criterio no solo de la distancia sino de la recursividad de una ruta (debido a que se debe también optimizar el zanjado y la compartición de ductos). El algoritmo modificado de Dijkstra modifica la matriz de costos en cada iteración disminuyendo el costo de las rutas más recursi- 
vas (haciéndolas más favorables en cada iteración). La Figura 8 muestra el trabajo de estas funciones.

- Evaluate cost: Esta función evalúa el costo del despliegue de las PON en la región bajo estudio, incluyendo todas las componentes especificadas en la Eq. (1), utilizando para el efecto costos actualizados y realistas obtenidos directamente de operadores y vendedores. El costo calculado por esta función es enviado a la PF para que lo utilice como criterio de decisión para decidir si la topología actual es menor (o no) que la que actualmente está registrada como la topología de menor costo.

\section{Resultados}

\section{A. Función primaria de OTS}

OTS encuentra una solución óptima de menor costo para el despliegue de múltiples PON en zonas urbanas extensas con muchos usuarios y con variadas demandas de ancho de banda. Dado que se trata de una solución basada en heurísticas no es seguro que se trata de la solución óptima absoluta, pero sí provee de una solución óptima cuya confiabilidad depende del número de iteraciones, de la precisció de la búsqueda y del tipo de barrido que se programe (muchos pasos de corta distancia, pero sin embargo ello implica más tiempo computacional consumido en la búsqueda de la solución).

OTS devuelve dos tipos de resultados: numéricos y gráficos. Los resultados numéricos incluyen tablas detalladas de la distribución de PON entre edificios y usuarios, como: a qué SSC y PSC están conectadas, la ubicación y dimensión de los SSC y PSC y la longitud y tipo de cables ópticos. Se presenta además información detallada de los costos, tanto de las componentes de cada subregión, así como de toda la zona evaluada.

Con el fin de presentar los resultados de un específico escenario de solución se ha escogido una región del centro de varias ciudades, escogiendo zonas que tengan potencialmente alrededor de 100000 usuarios. 


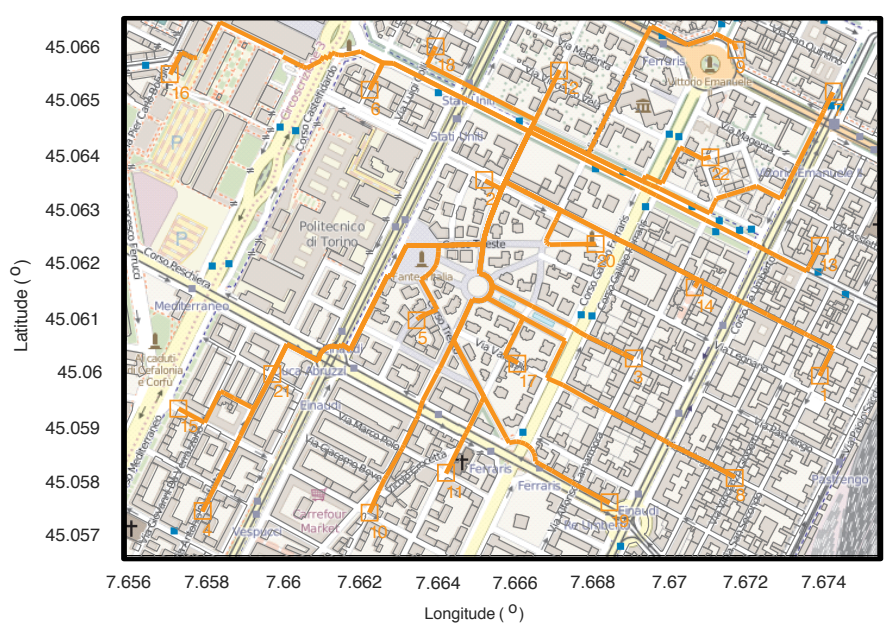

(a)

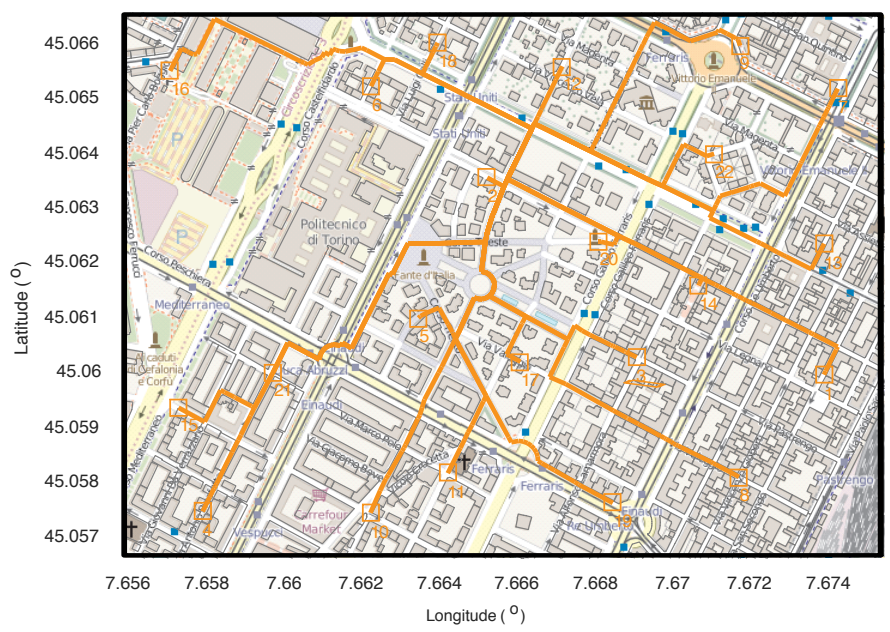

(b)

Figura 8. Función OF feeder: (a) Rutas desde la CO hasta los PSC generadas por la primera iteración de la función (usando una versión modificada de Dijkstra) (b) Rutas óptimas determinadas una vez que el algoritmo converge

En dichas zonas se corrió OTS para encontrar la solución de menor costo para el despliegue de todas las tecnologías PON especificadas en la Tabla 4. 
En la Figura 9 se muestra la solución encontrada por OTS en una porción de la ciudad de Turín (Italia). Se observa en las gráficas la posición de los PSC, los SSC, así como las rutas de los cables ópticos (de feeder y distribución). En la tabla VI se detalla los resultados de costos de implementación para las tecnologías PON consideradas y para cada uno de los seis bitrate especificados en la Tabla V.

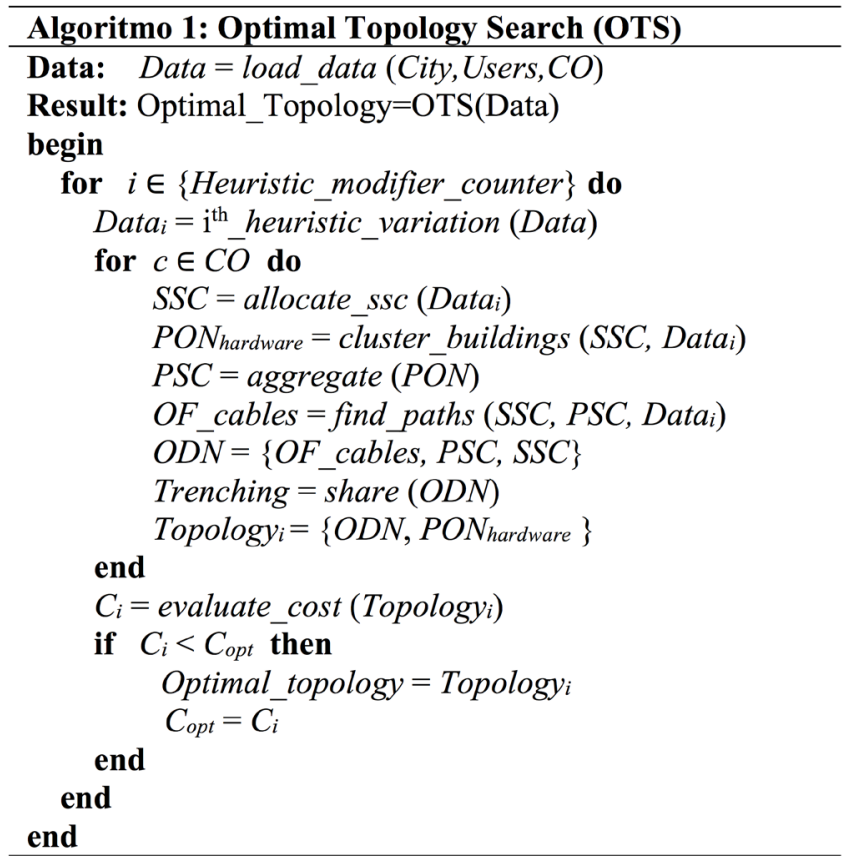

Tabla VI: Costo del despliegue para $2 \cdot 10^{5}$ usuarios

\begin{tabular}{|c|c|c|c|c|}
\hline \multirow{2}{*}{ Escenario } & \multicolumn{4}{|c|}{ COSTO (millones de USD) } \\
\cline { 2 - 5 } & GPON & XGPON & NGPON2 & UDWDM PON \\
\hline$\# 1$ & 51.4 & 82.2 & 113.3 & 157.1 \\
\hline$\# 2$ & 61.0 & 84.2 & 113.3 & 157.1 \\
\hline$\# 3$ & 106.6 & 93.3 & 113.3 & 157.1 \\
\hline$\# 4$ & 178.7 & 127.9 & 113.3 & 157.1 \\
\hline$\# 5$ & 394.6 & 207.8 & 146.8 & 159.9 \\
\hline$\# 6$ & - & 250.7 & 168.3 & 164.5 \\
\hline
\end{tabular}




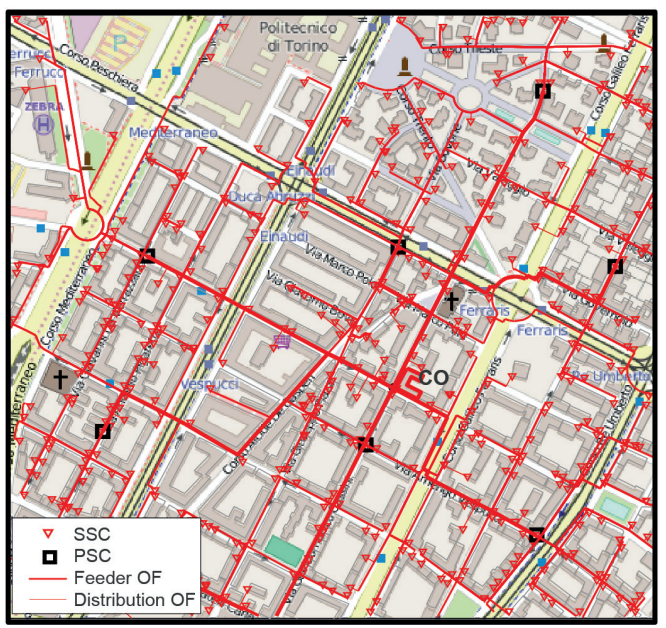

Figura 9. Resultado gráfico entregado por OTS: Una porción de una subregión de la ciudad de Turín, en la que se observa la ubicación óptima de los PSC, los

SSC asignados a cada edificio, y los cables ópticos enrutados en las calles

Nótese que una región con cerca de 100000 usuarios es realmente mucho más grande que la región del mapa de ejemplo mostrada en la Figura 9. En tal región, realmente se tienen varias subregiones servidas cada una de ellas por una CO diferente, con aproximadamente 20000 usuarios en cada subregión. En la Figura 10 se muestra un ejemplo de una zona urbana con tres CO (los edificios de cada subregión están pintados de un distinto color). Se trata solo de un ejemplo con fines ilustrativos ya que en la práctica las subregiones, con cerca de 20000 usuarios, son mucho más grandes.

En la Figura 11 se puede apreciar una comparación de los costos de implementación para las cuatro tecnologías PON consideradas en este estudio y para los seis escenarios de demanda de ancho de banda (bitrate) detallados en la Tabla V. Es interesante verificar que los resultados del escenario 1 (usuarios residenciales demandando entre 10 a $50 \mathrm{Mb} / \mathrm{s}$ de ancho de banda), que corresponde a un escenario de demanda real en el presente, da como resultado valores bien conocidos en la actualidad: GPON es la tecnología más redituable, ya que su costo de despliegue corresponde a aproximadamente $\$ 500$ por cada usuario (un costo absolutamente real hoy 
en día para el despliegue de GPON), mientras que XGPON es más costosa (no se justifica la inversión aún) y mucho más costosas en este escenario son la NGPON2 y la UDWDM PON.

En el escenario 2 (usuarios residenciales demandando entre 50 a $100 \mathrm{Mb} / \mathrm{s}$ ), escenario cercano al actual (real en algunas ciudades del primer mundo y casi real en la mayoría de las urbes), GPON se convierte en una solución un poco más costosa, sin embargo, las otras tres tecnologías siguen siendo más caras de implementar (nótese de todos modos que la brecha de costos se ha reducido pues no ha cambiado el precio de implementación de las otras tecnologías, solo ha subido el costo de GPON).

$\mathrm{El}$ escenario 3 (usuarios residenciales demandando entre $100 \mathrm{a}$ $400 \mathrm{Mb} / \mathrm{s}$ ), que es un escenario a corto plazo (pocos años en el futuro), implementar la tecnología GPON deja de ser la mejor opción ya que se torna más costosa que la XGPON, NGPON2 es no mucho más costosa que GPON y XGPON, lo cual es muy interesante dada la considerablemente mayor escalabilidad de NGPON2 frente a las otras dos.

Para el escenario 4 (usuarios residenciales demandando entre $100 \mathrm{Mb} / \mathrm{s}$ a $1 \mathrm{~Gb} / \mathrm{s}$ ), el cual es por supuesto un escenario a largo plazo (sobre todo por la consideración de tener usuarios que demanden en sus casas velocidades cercanas o iguales a $1 \mathrm{~Gb} / \mathrm{s}$ ), NGPON2 es claramente la mejor opción frente a las otras tecnologías. Se trata de un punto de inflexión que hace pensar en la conveniencia de implementar esta tecnología, ya estandarizada, incluso desde el escenario anterior.

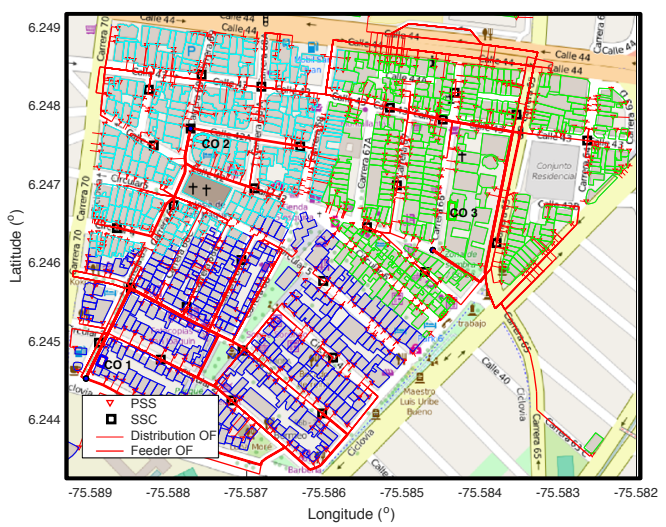

Figura 10. Resultado gráfico entregado por OTS: Una zona de la ciudad de Medellín, Colombia, con tres subregiones 


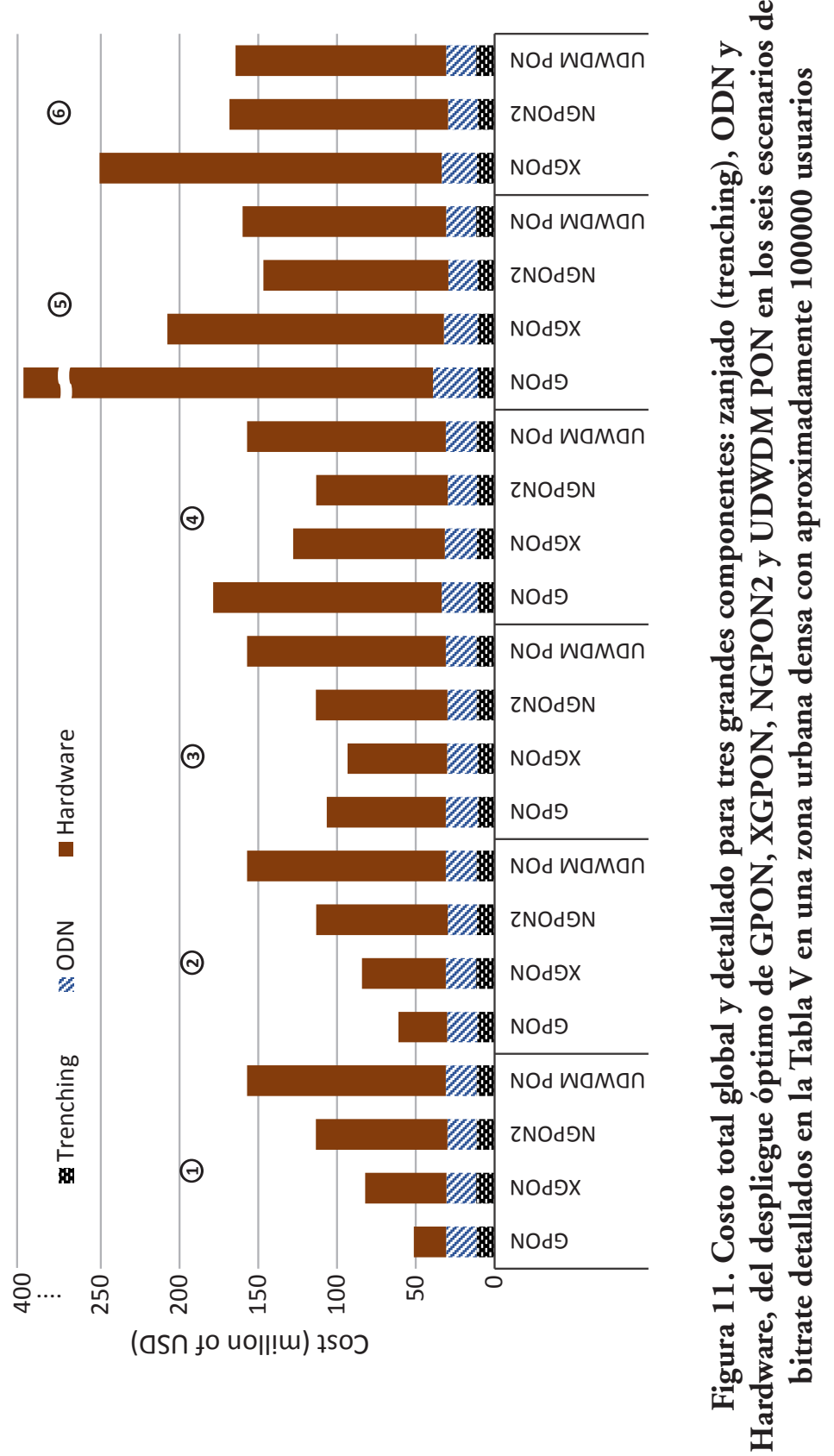


Los escenarios 5 y 6 son escenarios a muy largo plazo (el 6 más demandante que el 5 dado que pretende que los usuarios requieran al menos $1 \mathrm{G} / \mathrm{s}$, mientras que el 5 supone una demanda desde los $500 \mathrm{Mb} / \mathrm{s}$ ). En el escenario 6 el aumento de costo de GPON es tan alto que incluso no es práctico incluir a esa tecnología en la gráfica para dicho escenario. Es poco predecible en cuántos años en el futuro los usuarios podrían demandar estos enormes anchos de banda, pero desde este análisis queda claro que solo entonces tendría sentido pensar en implementar una tecnología tan costosa como lo sería la UDWDM PON (aunque es la que potencialmente puede entregar más ancho de banda a los usuarios en un futuro aparentemente lejano).

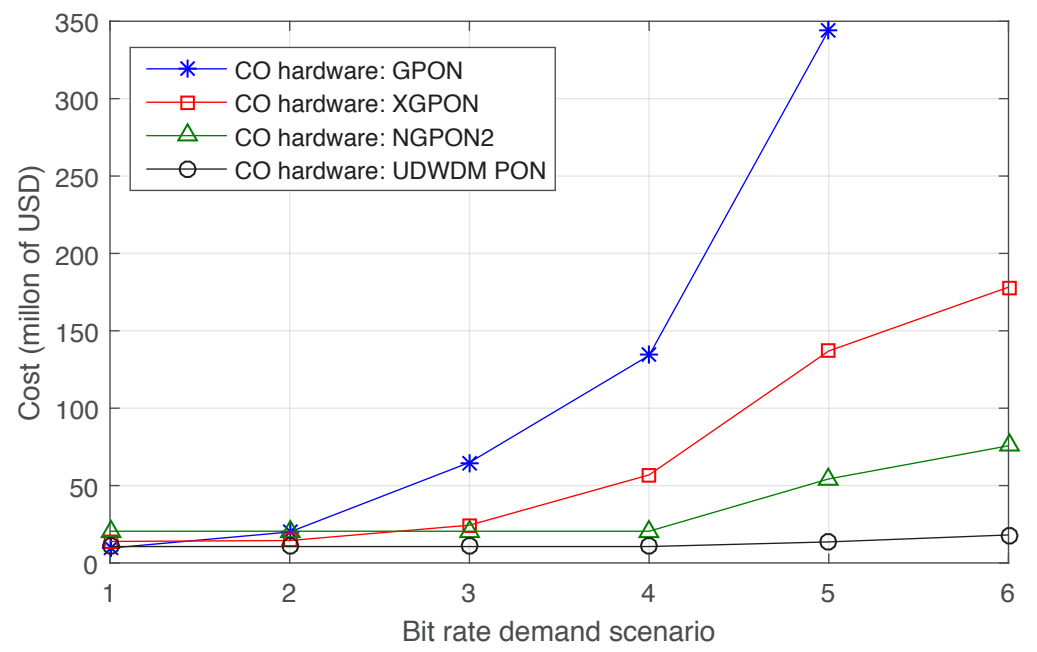

Figura 12. Costo total para aproximadamente 1000 usuarios del hardware PON en la CO para GPON, XGPON, NGPON2 y UDWDM PON en los seis escenarios de demanda de bitrate

La Figura 12 permite apreciar algo que en la figura anterior es también evidente: el aumento en el costo de implementación de una tecnología, con el aumento del ancho de banda, se debe principalmente al aumento en el costo del hardware PON, sobre todo en la CO. Esto se debe a que cuando una demanda de ancho de banda, por parte de 
los usuarios, comienza a sobrepasar los límites de una tecnología, esta comienza a convertirse en una solución costosa y poco redituable. Es importante ver en la figura que las tecnologías que casi no cambian su costo de implementación (aunque inicialmente mayor) son NGPON2 y UDWDM PON (esta última casi no cambia de costo de hardware incluso en el escenario 6, lo que significa que es la solución más económicamente escalable de todas). Las otras dos, GPON y XGPON, incrementan su costo exponencialmente en los seis escenarios considerados.

\section{Conclusiones}

El presente trabajo de investigación evidencia la utilidad, versatilidad y confiabilidad de OTS para el análisis tecno-económico del despliegue óptimo de redes PON en zonas urbanas extensas con heterogéneas y cambiantes demandas de ancho de banda por parte de los usuarios.

OTS puede ser utilizado para el análisis del despliegue óptimo no solo de PON, sino de todo tipo de red de información, como enlaces punto a punto o fronthauling para redes inalámbricas de nueva generación, lo que constituye potencialmente nuevos trabajos de investigación utilizando esta herramienta.

Se pueden llegar a conclusiones muy relevantes gracias al empleo de OTS en un análisis tecno-económico, que soporten la toma de decisiones de inversión, tales como los escenarios en los cuales el despliegue de una determinada tecnología sería o no redituable y el dimensionamiento de la escalabilidad de una solución tecnológica frente al cambio de las demandas de los usuarios.

\section{Referencias}

[1] E. Wong, "Next-Generation Broadband Access Networks and Technologies," J. Light. Technol., vol. 30, no. 4, pp. 597-608, Feb. 2012.

[2] Q. Guo and A. V. Tran, "20-Gb/s Single-Feeder WDM-PON Using Partial-Response Maximum Likelihood Equalizer," IEEE Photonics Technol. Lett., vol. 23, no. 23, pp. 1802-1804, Dec. 2011.

[3] G. Arévalo and D. Cárdenas, "Advantages of the use of VCSEL over RSOA for uplink transmission on WDM-PON networks," in 2012 IEEE Latin-America Conference on Communications, LATINCOM 2012 - Conference Proceedings, 2012. 
[4] K. Y. Cho, S. Member, U. H. Hong, M. Suzuki, and Y. C. Chung, "103-Gb / s Long-Reach WDM PON Implemented by Using Directly Modulated RSOAs," IEEE Photonics Technol. Lett., vol. 24, no. 3, pp. 2011-2013, 2012.

[5] J. Prat, "Technologies for a Cost Effective UDWDM-PON," in Procceedings of OFC, 2015, pp. 1-3.

[6] R. Gu, X. Liu, H. Li, and L. Bai, "Evolutional algorithm based cascade long reach Passive Optical Networks planning," China Commun., vol. 10, no. 4, pp. 59-69, Apr. 2013.

[7] L. Gouveia, M. J. Lopes, and A. de Sousa, "Single PON network design with unconstrained splitting stages," Eur. J. Oper. Res., vol. 240, pp. 361-371, Jul. 2014.

[8] J. Z. J. Zhang and N. Ansari, "Minimizing the Arrayed Waveguide Grating Cost and the Optical Cable Cost in Deploying WDM Passive Optical Networks," IEEE/OSA J. Opt. Commun. Netw., vol. 1, no. 5, pp. 352-365, Oct. 2009.

[9] R. Chowdhury and B. Jaumard, "A cross layer optimization scheme for WDM PON network design and dimensioning," 2012 IEEE Int. Conf. Commun., pp. 3110-3115, Jun. 2012.

[10] J. Segarra, V. Sales, V. Polo, and J. Prat, "Dimensioning OLT Architectures for UDWDM-PONs Employing Coherent Transceivers," in ICTON 2015: 17th International Conference on Transparent Optical Networks, 2015, no. Ga 318515, pp. 2-7.

[11] D. Nesset and S. Member, "NG-PON2 Technology and Standards," J. Ligthwave Technol., vol. 33, no. 5, pp. 1136-1143, 2015.

[12] D. L. Truong, P. T. Do, and A. T. Pham, "Optimization of Survivable Mesh LongReach Hybrid WDM-TDM PONs," J. Opt. Commun. Networking, OSA Publ., vol. 6, no. 1, pp. 62-76, 2014.

[13] O. Kipouridis, C. M. Machuca, A. Autenrieth, and K. Grobe, "Street-aware infrastructure planning tool for Next Generation Optical Access networks," in 6th International Conference on Optical Network Design and Modeling (ONDM), 2012, pp. 1-6.

[14] S. Pal, C. Zukowski, A. Nag, D. B. Payne, and M. Ruffini, "Cable Length Minimisation in Long-Reach-PON Planning for Sparsely Populated Areas," in International Conference on Optical Network Design and Modeling, 2014, pp. 19-22.

[15] G. V. Arévalo, R. C. Hincapié, and J. E. Sierra, "Optimization model for UDWDM-PON deployment based on physical restrictions and asymmetric users' clustering," in Proc. SPIE 9626, Optical Systems Design 2015: Optical Design and Engineering VI, 2015, vol. 9626, pp. 1-11.

[16] A. Eira, J. Pedro, and J. Pires, "Optimized Design of Multistage Passive Optical Networks," J. Opt. Commun. Networking, OSA Publ., vol. 4, no. 5, pp. 402-411, 2012. 
[17] G. V. Arévalo, J. E. Sierra, R. C. Hincapié, and R. Gaudino, "A novel algorithm for PON optimal deployment over real city maps and large number of users," in IET Conference Publications, 2016, vol. 2016, no. CP704.

[18] G. V. Arévalo, R. C. Hincapié, and R. Gaudino, "Optimization of multiple PON deployment costs and comparison between GPON, XGPON, NGPON2 and UDWDM PON," Opt. Switch. Netw., vol. 25, 2017.

[19] ITU-T, "G.984.1 Gigabit-capable passive optical networks (GPON): General characteristics," 2008.

[20] R. Murano, "Optical Component Technology Options for NGPON2 Systems," OFC2014, no. c, pp. 3-5, 2014.

[21] H. Rohde, E. Gottwald, A. Teixeira, J. D. Reis, A. Shahpari, K. Pulverer, and J. S. Wey, "Coherent Ultra Dense WDM Technology for Next Generation Optical Metro and Access Networks," J. Light. Technol., vol. 32, no. 10, pp. 2041-2052, 2014.

[22] H. Rohde, S. Member, E. Gottwald, E. Weis, P. Wagner, Y. Babenko, D. Fritzsche, and H. Chaouch, "Trials of a Coherent UDWDM PON Over Field-Deployed Fiber: Real-Time LTE Backhauling, Legacy and 100G Coexistence," J. Ligthwave Technol., vol. 33, no. 8, pp. 1644-1649, 2015.

[23] M. R. Garey, R. L. Graham, and D. S. Johnson, "The Complexity of Computing Steiner Minimal Trees," SIAM J. Appl. Math., vol. 32, no. 4, pp. 835-859, 1977.

[24] M. De Berg, M. Van Kreveld, and M. Overmars, "Computational geometry: algorithms and applications," Springer-Verlag, New York, vol. 3ra.ed., no. ISBN: 978-3-540-77973-5, pp. 191-218, 2008.

[25] F. Glover, M. Laguna, and R. Marti, "Principles of tabu search," Approx. Algorithms Metaheuristics, vol. 23, pp. 1-12, 2007.

[26] L. X. L. Xia, J. S.-Y. J. Sheng-Yi, and S. X.-K. S. Xiao-Ke, "A Novel Fast Clustering Algorithm," in International Conference on Artificial Intelligence and Computational Intelligence, 2009, vol. 4, pp. 284-288.

\section{Sobre los autores}

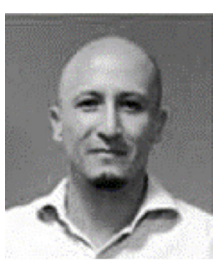

Germán V. Arévalo recibió su título de ingeniería en Electrónica por parte de la Escuela Politécnica Nacional, Quito - Ecuador, en el 2003. Recibió su título de Máster en Ciencias en Comunicaciones Ópticas por parte del Politécnico di Torino, Turín - Italia, en el 2004 y su título de $\mathrm{PhD}$, por parte de la Universidad Pontificia Bolivariana de Medellín - Colombia, en el 2016. Actualmente es profesor titular principal y director de la Carrera de Ingeniería en Telecomunicaciones y del programa de Maestría de Investigación en Telemática, en la Universidad Politécnica Salesiana (UPS) del Ecuador. Es a su vez Coordinador del Grupo de Investigación en Electrónica y Telemática, GIETEC, de la UPS. Su investigación se ha desarrollado alrededor de las redes ópticas de nueva generación, a nivel experimental y teórico. 


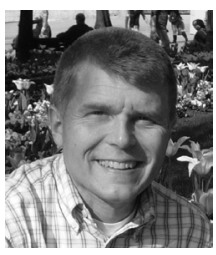

Roberto Gaudino recibió su título de ingeniería en Electrónica, con Summa Cum Laude, por parte del Politécnico di Torino (POLITO), Turín - Italia, en 1993 y su PhD en el POLITO, en 1998. Es actualmente profesor asociado del POLITO. Ha dirigido en el pasado proyectos colaborativos con TIM Italia, que han significado contribuciones al nuevo estándar de redes PON, NGPON2 y hacia el estándar futuro de PON (conocido hoy como HS-PON). Ha sido coordinador de varios proyectos europeos, como el POF-ALL y el POF-PLUS, enfocados en redes ópticas plásticas de bajo costo. Participó en importantes proyectos como el ALPHA y el E-PhotonOne (donde actuó como coordinador del WP en "Comunicaciones de Corto Alcance"). Además, se ha desempeñado como director del programa de Ingeniería en Telecomunicaciones del POLITO y actualmente es el coordinador científico del PhotoNext Center, que se enfoca en comunicaciones ópticas de ultra-alta velocidad sensores ópticos y componentes para aplicaciones industriales. PhotoNext constituye uno de los 11 más grandes centros departamentales del POLITO, cuyo financiamiento comenzó en el 2017. 